\title{
MODELLING THE POSITION OF CELL PROFILES ALLOWING FOR BOTH INHOMOGENEITY AND INTERACTION
}

\author{
LINDA STOUGAARD NiELSEN \\ Laboratory for Computational Stochastics, Department of Mathematical Sciences, University of Aarhus, \\ Denmark. Email: lins@imf.au.dk. \\ (Accepted October 6, 2000)
}

\begin{abstract}
It is of interest to consider models for point processes that allow for interaction between the points as well as for inhomogeneity in the intensity of the points. Markov point process models are very useful to describe point interaction and can also be used to describe inhomogeneity. A particular type of inhomogeneous Markov point processes obtained by transforming a homogeneous Markov point process will be considered. The position of cell proles in a $2 \mathrm{D}$ section of the mucous membrane in the stomach of a rat will be examined using this model.
\end{abstract}

Keywords: Inhomogeneity, Interaction, Likelihood, Markov point process, Poisson point process, Strauss point process.

\section{INTRODUCTION}

The position of the centres of cells in tissue can be described by a spatial point process, where each cell centre is represented by a point. A point process is a stochastic model for point patterns that describes the properties of the point pattern: Trend in the intensity of the points (inhomogeneity) and interaction between the points.

In surface covering layers (epithelium) such as skin, mucous membranes and cartilage, a gradient can usually be observed in the direction perpendicular to the surface. In planes parallel to the surface homogeneity can be assumed. Thus, it is of interest to consider models for point processes that allow for inhomogeneity in a given direction. Furthermore, such models must be able to describe interaction between points. Between the cell centres there is a natural inhibition since a cell has an extend and two cell centres therefore have to be a certain distance apart. Considering cell division, inhibition on a small scale and clustering on a larger scale might be observed.

Typically we do not observe the full 3 dimensional data but only a 2 dimensional section where the trend is along one of the two dimensions. In this paper we will consider a 2 dimensional section of epithelium and model the position of cell proles using a point process with a directional gradient and small scale inhibition.

Markov point process models are e xible and tractable models for describing interaction. Homogeneous Markov point processes are well studied and tools for statistical inference well developed, see e.g. Diggle (1983), Geyer (1999) and
Baddeley and Turner (1998). Inhomogeneous Markov point processes have been much less considered.

The model proposed by Jensen and Nielsen (2000) is an inhomogeneous Markov point process model, where the inhomogeneity is obtained by applying a bijective differentiable mapping to a homogeneous Markov point process. The interaction is then inherited from the homogeneous Markov model. In cases of inhibition between the points there is strong experimental evidence that the statistical inference can be separated into the statistical inference for a inhomogeneous Poisson point process (model with no interaction) and a homogeneous Markov point process, cf. Jensen and Nielsen (2000). However, this issue still needs a thorough investigation. In this paper we will use this type of statistical inference and will see that it gives a good description of the epithelium data.

\section{MATERIAL AND METHODS}

The image in Figure 1 was supplied by Thomas F. Bendtsen. The image shows a $2 \mathrm{D}$ section of the mucous membrane of the stomach of a healthy rat, where the darker spots are the cell proles. In the top of the image we have the cavity of the stomach, and in the bottom of the image the muscle tissue begins. In this paper the proles only in the marked region are studied. In right hand-side of Figure 1 the position of the nuclei in this region are shown. For convenience, the window is scaled into the window $[0,893] \times[0,1]$. In what follows, we nd a model that describe this point pattern.

As mentioned in the introduction there is an 
obvious directional trend in the point pattern and small scale inhibition since two cells cannot overlap. In the remains of this paper, the point pattern will be analyzed using the class of Markov point processes by transformation, which will be introduced below.

Let $X$ denote a 2 dimensional homogeneous Markov point process, and let $\gamma$ be the parameter that describes the point interaction. In Markov point processes, interactions are only allowed between neighbour points. A typical example of a Markov point process is the Strauss point process, cf. Strauss (1975), where two points are neighbours if their distance is less than some constant $R>0$. The probability density of the point process is controlled by $\gamma^{s(x)}$, where $s(x)$ is the number of neighbours in a given point pattern $x$. Then, if $0<\gamma<1$, the tendency of points rejecting each other increases the smaller $\gamma$ is. A value of $\gamma$ equal to 0 is the hard-core model where no pair of points have distance less than $R$, and $\gamma=1$ is the Poisson model, the model with no interaction at all.

Let $h_{\theta}(\eta, \xi)=\left(\eta, l_{\theta}(\xi)\right)$ be a differentiable bijective parametrized mapping. Then, according to Jensen and Nielsen (2000), $Y=h_{\theta}(X)$ is a Markov point process, inhomogeneous in the direction of the second coordinate, and with density

$$
f_{Y}(y)=f_{X}\left(h_{\theta}^{-1}(y)\right) \prod_{i=1}^{n(y)}\left(l_{\theta}^{-1}\right)^{\prime}\left(y_{i 2}\right),
$$

where $f_{X}$ is the density of $X, y_{i 2}$ is the second coordinate of the $i$ 'th point $y_{i}$ of $y$ and $n(y)$ is the number of points in $y$. As $f_{X}$ controls the interaction between the points, the interaction between points in the transformed point process $Y$ is inherited from the homogeneous model $X$. The differential term $\left(l_{\theta}^{-1}\right)^{\prime}$ controls the inhomogeneity. For more detail, see Jensen and Nielsen (2000). Note that the rst coordinates are not transformed, and therefore the model (1) only describes inhomogeneity in the second coordinate direction.

In applications such as the point pattern from Figure 1, we have an inhomogeneous point pattern which we want to describe by a point process model. Using the transformation approach, we assume that the point pattern in question, say $y$, is a realization of a transformation-inhomogeneous point process $Y$. The aim is then to nd a transformation such that the back-transformed point pattern $x=h_{\theta}^{-1}(y)$ is a homogeneous point pattern. Finally, well-known theory for homogeneous point patterns can be applied in order to nd a model $X$ describing $x$.

The problem of nding a suitable transformation is not simple. The density (1) can be split into the density for a homogeneous point process and that of an inhomogeneous Poisson point process. However, the inhomogeneity parameter $\theta$ enters into both parts, and so maximum likelihood estimation of $\theta$ is not based on the inhomogeneous Poisson part alone. In practice however (simulation studies), the estimate $\hat{\theta}_{0}$ based on the inhomogeneous Poisson point process seems to be very good to describe the inhomogeneity. Thus, $\hat{\theta}_{0}$ will here be used as an estimate of $\theta$.

In order to analyze a homogeneous point pattern
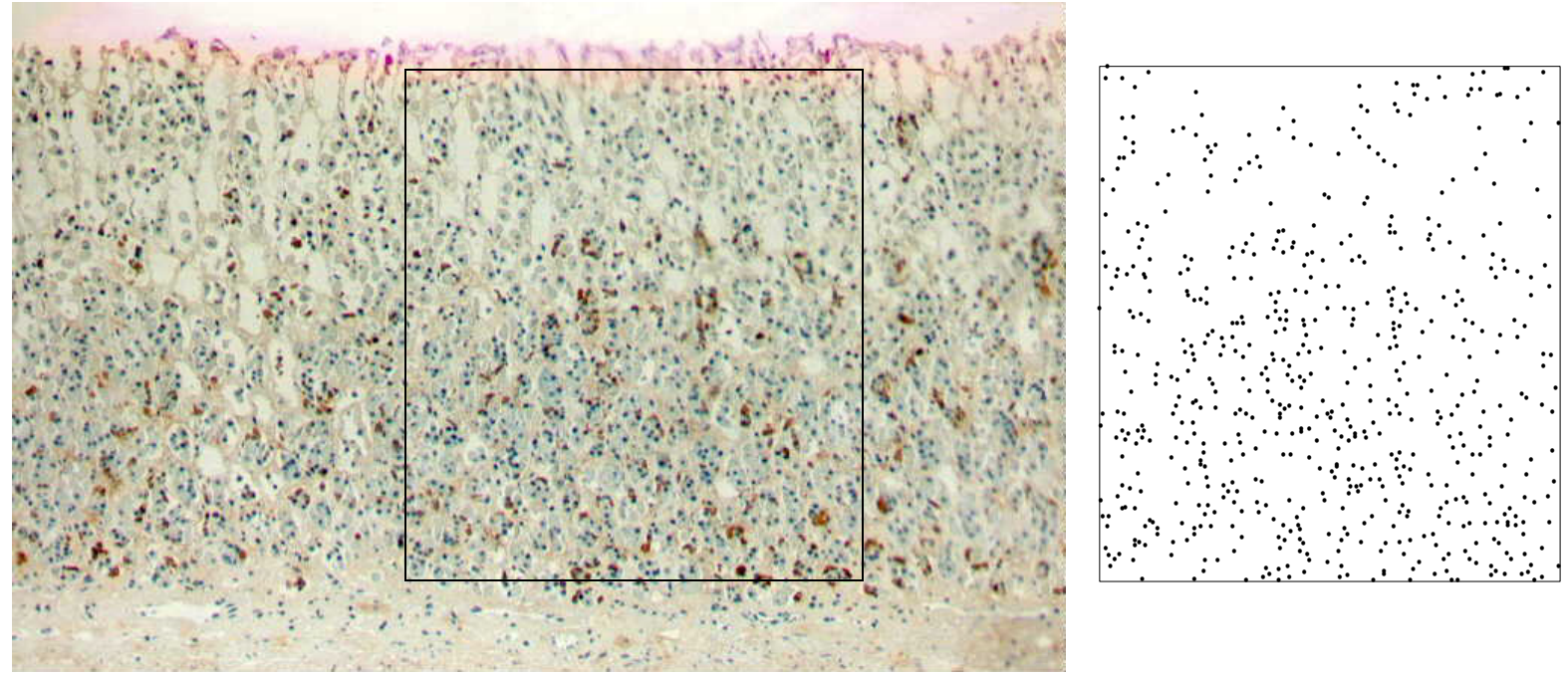

Fig. 1. Image by Thomas F. Bendtsen showing a 2D section of the mucous membrane in the stomach of a healthy rat. To the right, the positions of the nuclei of the cell proles in the marked region of the image are plotted. This is the point pattern considered in the present paper. 
we first consider summary statistics in order to find the characteristics of the particular point pattern such that a suitable parametric model can be proposed, see e.g. Diggle (1983). Then the parameters in the proposed Markov point process model are estimated using likelihood or pseudo-likelihood theory, see Geyer (1999) and Baddeley and Turner (1998). Notice that it is also possible to use likelihood and pseudolikelihood theory in the full inhomogeneous model. This is however much more complicated, see Jensen and Nielsen (2000).

\section{RESULTS}

To describe the inhomogeneity in the second coordinate direction of the point pattern from Figure 1, we will consider exponential inhomogeneity, i.e.

$$
\left(l_{\theta}^{-1}\right)^{\prime}(\eta) \propto \mathrm{e}^{\theta \eta} .
$$

The following mapping $l_{\theta}:[0,1] \rightarrow[0,1]$ has the above property,

$$
l_{\theta}(\eta)=\frac{1}{\theta} \log \left(\eta\left(\mathrm{e}^{\theta}-1\right)+1\right) .
$$

In Figure 2 (b) the back-transformed data point pattern is shown, where the inhomogeneity parameter used in the transformation is the maximum likelihood estimate $\hat{\theta}_{0}$ in the inhomogeneous Poisson model. Notice that this point pattern appears to be homogeneous.

To determine the interaction structure of a homogeneous point pattern, we compare the point pattern with a homogeneous Poisson point process. This is typically done by comparing the $F, G, J$, $K$ and pair-correlation functions. See e.g. Diggle (1983), Stoyan et al. (1995) and van Lieshout and Baddeley (1996) for more details on these functions. In what follows, I have considered only the $F$ and $G$ functions, but the conclusions would be the same using any of the other functions. In the first plot of Figure 3 (a) the $F$ function for the back-transformed data and for the homogeneous Poisson point process are plotted. The $F$-function can be thought of as a statistic that measures the amount of empty space in the point pattern. As seen, the back-transformed point pattern has the same amount of empty space as the Poisson point process. In the second plot of Figure 3 (a), the $G$ function is shown. This function is the probability function for the nearest neighbour distances in the point pattern. As seen, the backtransformed point pattern has less of the small nearest neighbour distances than the Poisson point process, which is a sign of small scale inhibition. This is of course due to the fact that we only consider the centres of the cell profiles.

A Strauss point process is a useful model for describing inhibition. In Figure 3 (b) the data is compared with a Strauss point process with parameters $\beta=760, \gamma=0.09$ and $R=0.007$. Due to the very small distance $R$, the amount of empty space is close to that of the Poisson point process. The amount of very small nearest neighbour distances is reduced. From the two plots we conclude that the back-transformed

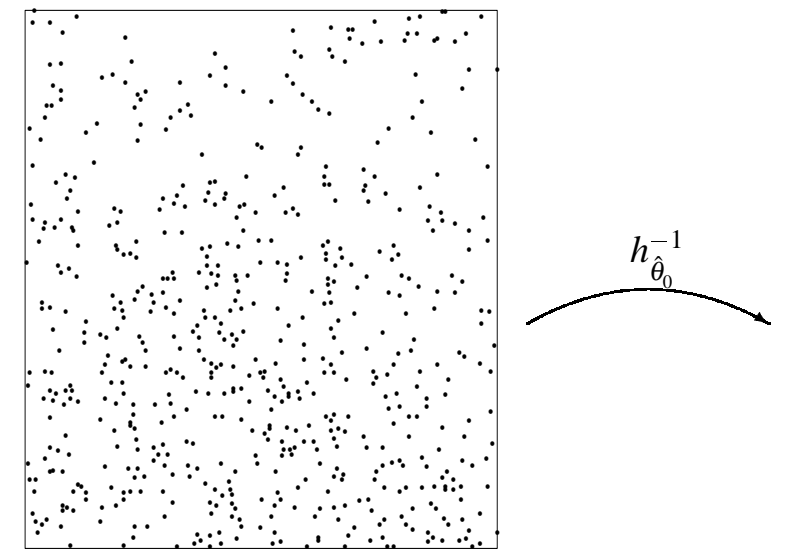

(a) Centres of cell profiles

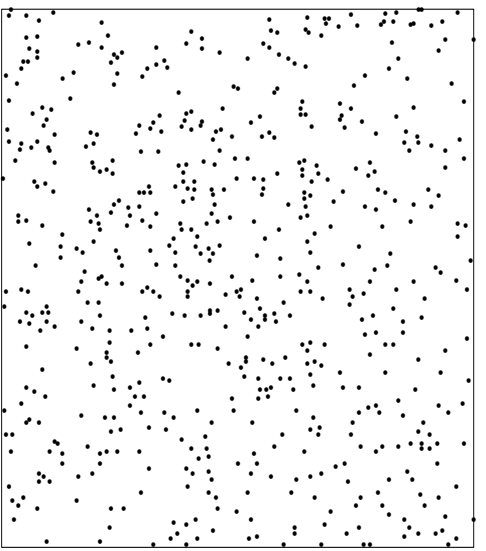

(b) Back-transformed centres

Fig. 2. Point pattern y from Figure 1 of cell profile centres (a) and the corresponding back-transformed point pattern $x=h_{\theta}^{-1}(y)(b)$. The transformation parameter is the maximum likelihood estimate in the inhomogeneous Poisson point process model, $\hat{\theta}_{0}=1.3043$. 

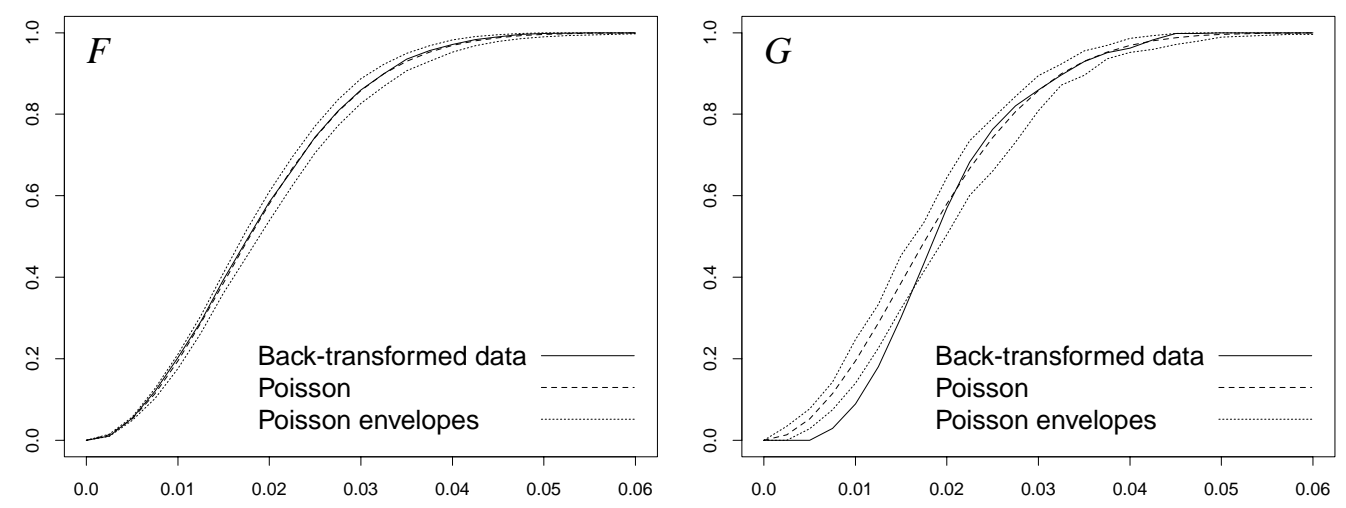

(a) Back-transformed data vs Poisson model
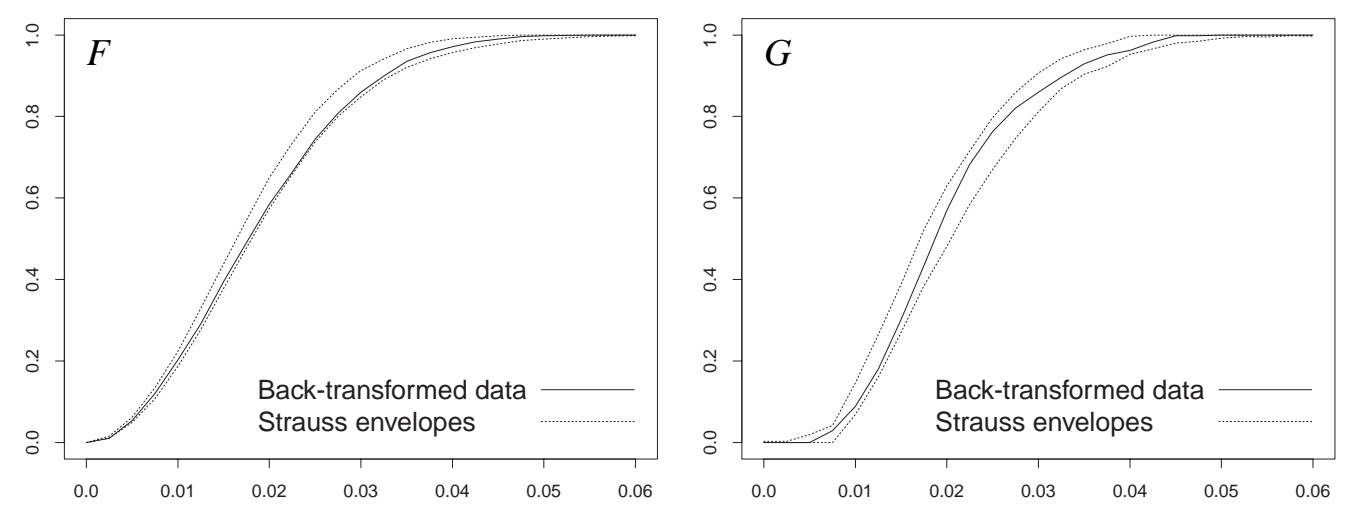

(b) Back-transformed data vs regular Strauss model

Fig. 3. Empty space function $F$ and nearest neighbour function $G$ for the back-transformed data point pattern versus the Poisson point process (a) and the Strauss point process with parameters $\beta=760, \gamma=0.09$ and $R=0.007$ (b). Envelopes are the pointwise maxima and minima of 39 simulated processes.

data point pattern can be assumed to be a realization of the Strauss point process with parameters as above. Hence, the data point pattern can be regarded a realization of the exponential inhomogeneous Strauss point process with density

$$
f_{Y}(y)=\alpha(\beta, \gamma) \beta^{n(y)} \gamma^{s\left(h_{\theta}^{-1}(y)\right)}\left(\frac{\theta}{\mathrm{e}^{\theta}-1}\right)^{n(y)} \prod_{i=1}^{n(y)} \mathrm{e}^{\theta y_{i 1}},
$$

where $\theta=1.3043, \beta=760, \gamma=0.09$ and $R=0.007$. In Figure 4 the data is plotted together with four simulations from this point process.

Note that, except for the small scale inhibition caused by non-overlapping cells, the data point pattern is very close to being an inhomogeneous Poisson point process, cf. Figure 3 (a). Thereby the estimate $\hat{\theta}_{0}$ based on the inhomogeneous Poisson model is a very accurate estimate.

\section{DISCUSSION}

It appears as if there is also a gradient in the rst coordinate of the data point pattern. The maximum likelihood estimate based on the inhomogeneous Poisson point process is $\hat{\theta}_{02}=-0.27159$. The estimate is very small and it could be interesting to perform a statistical test: $\theta_{2}=0$. More interesting would be to compare gradients within various sections from the same rat. Probably the gradient in the rst coordinate can be explained as variation, while the gradient in the second direction is signicant. It could also be interesting to compare different rats and to compare healthy rats with rats treated with anti-acid drugs.

Furthermore, I have not distinguished between the type of cells in the mucous membrane. There is much interest in a particular type of cells, the ECL cells, and it could be interesting to test whether these cells are uniformly distributed among all the cells. This is the 


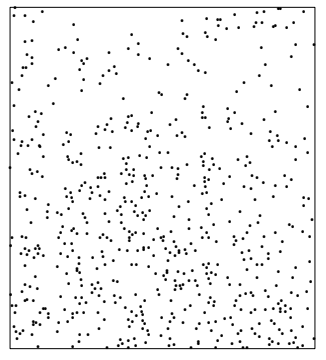

(a) Data
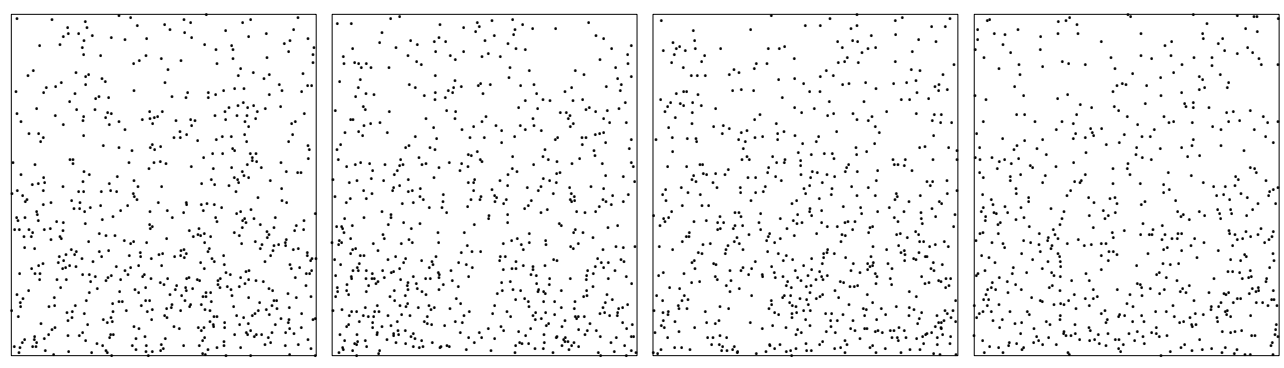

(b) Transformed simulations of regular Strauss point processes

Fig. 4. Plot of the data points (a) and four simulations from the exponential inhomogeneous Strauss point process (b).

case if the gradient for the ECL cells is the same as the gradient for all the cells.

Note that, in this paper we have modelled a 2D section of a 3D structure. Supposing that we have the full 3D data set, the same model could be used where now $X$ is a $3 \mathrm{D}$ homogeneous point process, the transformation is $h_{\theta}(\eta, \xi, v)=\left(\eta, l_{\theta}(\xi), v\right)$ and the inhomogeneous model is still of the form (1). However, we do not have information about the third dimension, and this will most often be the situation in reality. Conclusions about the model for the 3D data based on the model obtained from a 2D section is not straightforward. Methods used in Stereology may be useful. See Stoyan et al. (1995, Section 11.6.2) and Hahn et al. (1999).

Finally, it should be noticed that the transformation model only describes the position of the cell prole centres and not the whole image. Transforming the image would give homogeneous distributed positions but inhomogeneous distributed prole sizes. The image should be described by a marked point process, see e.g. Baddeley and Møller (1989), with prole positions modelled by (2) and marks of the points being the sizes and shapes of the cell proles.

A preliminary report of some of the data was presented at the Xth International Congress for Stereology, Melbourne, Australia, 1-4 November 1999.

\section{ACKNOWLEDGEMENTS}

I would like to thank Thomas F. Bendtsen for letting me use his data and Hans Jørgen G. Gundersen for bringing data to my attention. Furthermore, I thank both Thomas and Hans Jørgen for their effort in making me understand some of the biology behind the image.
I am also very grateful to my supervisor Eva B. Vedel Jensen, to Ute Hahn and to the anonymous referee for correcting errors and improving the paper.

\section{REFERENCES}

Baddeley AJ, Møller J (1989). Nearest-neighbour Markov point processes and random sets. Int Statist Rev 57:89121.

Baddeley AJ, Turner R (1998). Practical maximum pseudolikelihood for spatial point patterns. Australian and New Zeeland Journal of Statistics (in press).

Diggle PJ (1983). Statistical analysis of spatial point patterns. Academic Press.

Geyer CJ (1999). Likelihood inference for spatial processes. In: Barndorff-Nielsen OE, Kendall WS, van Lieshout MNM, eds. Stochastic Geometry, Likelihood and Computation. Chapman and Hall/CRC: Boca Raton.

Hahn U, Micheletti A, Pohlink R, Stoyan D, Wendrock H (1999). Stereological analysis and modelling of gradient structures. J Microsc 195:113-24.

Jensen EBV, Nielsen LS (2000). Inhomogeneous Markov point processes by transformation. To be published in Bernoulli.

Stoyan D, Kendall WS, Mecke J (1995). Stochastic geometry and its Applications. Chichester: Wiley \& sons, 2nd edition.

Strauss DJ (1975). A model for clustering. Biometrika 62:467-75.

van Lieshout MNM, Baddeley AJ (1996). A nonparametric measure of spatial interaction in point patterns. Stat Neerl 50:344-61. 